Questions vives

\section{Questions Vives}

Recherches en éducation

$N^{\circ} 27 \mid 2017$

(Re) découvrir John Dewey : quelle actualité en formation des adultes?

\title{
Équipements mobiles au collège : quelles appropriations par les élèves et les enseignants?
}

Le cas d'Ordival entre 2013 et 2015

Christelle Pauty-Combemorel et Georges-Louis Baron

\section{(2) OpenEdition \\ Journals}

\section{Édition électronique}

URL : http://journals.openedition.org/questionsvives/2119

DOI : 10.4000/questionsvives. 2119

ISSN : $1775-433 X$

Éditeur

Université Aix-Marseille (AMU)

\section{Édition imprimée}

Date de publication : 31 juillet 2017

ISBN : 978-2-912643-51-3

ISSN : 1635-4079

Référence électronique

Christelle Pauty-Combemorel et Georges-Louis Baron, «Équipements mobiles au collège : quelles appropriations par les élèves et les enseignants? », Questions Vives [En ligne], № 27 | 2017, mis en ligne le 31 décembre 2017, consulté le 20 avril 2019. URL : http://journals.openedition.org/ questionsvives/2119; DOI : 10.4000/questionsvives.2119

Ce document a été généré automatiquement le 20 avril 2019.

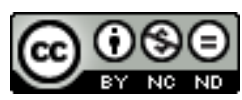

Questions Vives est mis à disposition selon les termes de la licence Creative Commons Attribution -

Pas d'Utilisation Commerciale - Pas de Modification 4.0 International. 


\section{Équipements mobiles au collège : quelles appropriations par les élèves et les enseignants?}

Le cas d'Ordival entre 2013 et 2015

Christelle Pauty-Combemorel et Georges-Louis Baron

\section{Introduction : les équipements en matériels informatiques au collège en France}

1 Différentes opérations relevant d'initiatives politiques ont été lancées en France dès le début des années 2000 dans le but de réduire les fractures numériques et sociales : six conseils départementaux ont, en effet, équipé les collégiens et leurs enseignants en matériels informatiques. En 2001, les collégiens landais ont reçu des ordinateurs portables (Jaillet, 2004; Khaneboubi, 2007), puis ceux des Bouches-du-Rhône en 2003 (Liautard, 2007), d'Ille-et-Vilaine en 2004 (J.-L. Rinaudo, Turban, Delande, \& Ohana, 2008), de Corrèze (IGEN, 2011), de l'Oise en 2008 (Khaneboubi, 2010), et enfin du Val-de-Marne en 2012 (Baron \& Combemorel-Pauty, 2015; Baron, Voulgre, Khaneboubi, Zablot, \& Combemorel-Pauty, 2014). Ces plans d'équipements en matériels informatiques préludent les objectifs nationaux annoncés en 2014 par le Président de la République notamment par la dotation à chaque élève de $5^{e}$ d'une tablette numérique (Battaglia, 2014).

2 En s'intéressant aux opérations de dotation massive, Khaneboubi (2009a) a identifié plusieurs caractéristiques communes. Les objectifs communs affichés par les collectivités territoriales porteuses des projets consistent à équiper individuellement chaque collégien avec un ordinateur portable ou une tablette numérique pour leur permettre d'avoir accès aux Technologies de l'Information et de la Communication (TIC), et ce pour favoriser, dans une visée égalitariste, la réussite scolaire de tous les élèves. Si ces plans sont souvent considérés comme des objets «magiques » dans les discours des décideurs, les recherches portant sur les différentes opérations d'équipements montrent que les machines sont 
finalement peu employées à des fins scolaires par les élèves et leurs enseignants (Khaneboubi, 2009b ; Plantard, 2015 ; Rinaudo, 2007).

Dans cet article, nous cherchons à comprendre de quelles manières les acteurs bénéficiant d'équipements informatiques distribués par le conseil départemental du Valde-Marne se sont appropriés ces matériels entre 2013 et 2015. Qu'est-ce qui favorise ou freine leur appropriation? Quelles sont les évolutions perceptibles durant cette période?

4 Après avoir présenté le contexte dans lequel nous avons réalisé notre recherche ainsi que le cadre réflexif que nous avons mobilisé, nous présenterons nos principaux résultats sur les effets de l'introduction des équipements dans les établissements scolaires observés en fonction des pôles du modèle PADI (Wallet, 2010). Nous proposerons, enfin, des éléments de discussion.

\section{L'opération Ordival}

5 Depuis la rentrée 2012, une opération massive visant à équiper chaque collégien du Valde-Marne en ordinateurs portables a été mise en œuvre à l'initiative du conseil départemental en partenariat avec le rectorat de l'académie de Créteil. Tous les élèves entrant dans l'un des cent quatre collèges publics et des vingt-trois privés sous contrat ont reçu un ordinateur portable nommé «Ordival » lors de leur entrée en sixième pour un usage en classe et à la maison. Ils ont ensuite pu en bénéficier jusqu'à la fin de leur troisième. Quatre mille enseignants sont également concernés ${ }^{1}$. Aujourd'hui, les collégiens des quatre niveaux sont équipés.

6 Ces machines, dont le système d'exploitation est Windows Professionnel pour l'éducation ${ }^{2}$, sont équipées d'un accès à une médiathèque numérique ${ }^{3}$ constituée de ressources pédagogiques et d'applications mobiles rassemblées par le pôle numérique de l'académie de Créteil, produit d'un partenariat entre l'éducation nationale et le conseil départemental regroupant la délégation académique au numérique éducatif (DANE), le réseau de Création et d'accompagnement pédagogique (CANOPÉ) et le Centre de liaison de l'enseignement et des médias d'information (CLEMI). Les élèves et les enseignants ont donc la possibilité de télécharger via la médiathèque à ces ressources ou d'accéder à des manuels numériques disponibles sur les ordinateurs portables. Des logiciels de type bureautiques (suite Libreoffice), graphiques (GIMP, Géogébra, etc.), documentaires (dictionnaire Le Robert) ou multimédia (Windows Movie Macker, Audacity, etc.) y sont accessibles.

7 Afin de favoriser l'appropriation de ces matériels informatiques par les enseignants, le pôle numérique, sous l'autorité du rectorat, propose d'accompagner ces derniers à la prise en main des ordinateurs et de leurs ressources. Plusieurs solutions sont mises en avant par le pôle. Des formations sont proposées dans les établissements scolaires ou à l'extérieur sur la découverte de l'ordinateur et de ses fonctionnalités, l'utilisation des ressources numériques implémentées dans la machine ou encore l'emploi du vidéoprojecteur interactif avec l'Ordival. Des réunions ont également été organisées au sein des collèges à l'initiative des chefs d'établissement ou à la demande des enseignants pour permettre à ces derniers de mutualiser leurs pratiques ou de requérir l'assistance d'une personne-ressource. Enfin, des ressources numériques (cours en ligne, animation sur You Tube, fiches pédagogiques appelées "Médiafiches ») sont aussi suggérées aux enseignants par les acteurs du pôle via leur site internet ou leurs comptes Twitter pour 
leur offrir la possibilité, s'ils le souhaitent, de s'auto-former en y accédant de façon autonome.

\section{Cadre réflexif}

Pour mieux comprendre comment les élèves et les enseignants s'approprient les ordinateurs portables, nous nous sommes appuyés sur le "modèle PADI " proposé par Wallet (2010). Ce dernier considère la possibilité d'étudier l'introduction des technologies en éducation de manière systémique à partir de quatre entités (Pédagogie, Acteurs/ Agents didactiques, Dispositif, Institution) interdépendantes liées par des liens bidirectionnels. Nous proposons de définir chacun de ces pôles de la manière suivante :

- le pôle Pédagogie positionne les acteurs du système scolaire face à leur « conception de l'enseignement et de l'apprentissage » (Voulgre, 2011, p. 27);

- le pôle Acteurs/Agents didactiques est composé dans un établissement de toutes les personnes dont les missions et les statuts sont codifiés et réglementés par Le code de l'Éducation. Il regroupe autant les formateurs que les formés. Ces acteurs sont reliés les uns aux autres et peuvent avoir "des effets positifs ou négatifs" sur l'équilibre de l'établissement (Voulgre, 2011, p. 26);

- le pôle Dispositif revoie à une réalité assez hétérogène puisqu'il permet d'aborder aussi bien les organisations que les personnes ou les instruments. Dans ce sens, Peraya définit un dispositif comme « une instance, un lieu social d'interaction et de coopération possédant ses intentions, son fonctionnement matériel et symbolique enfin, ses modes d'interaction propre» (Peraya, 1999, p. 153);

- le pôle Institution a pour mission d'instituer en régulant ou en administrant, c'est-à-dire en rendant officiels les règles et les modes de fonctionnements au plan national (Ministère de l'Éducation nationale), régional (académie de Créteil) ou départemental (conseil départemental du Val-de-Marne).

Dans cette approche, introduire une technologie telle qu'Ordival a des effets sur l'ensemble des pôles, le système tendant à conserver un état d'équilibre (homéostasie). Ce type de modélisation a une valeur heuristique et peut permettre de questionner les relations et les tensions liées à l'introduction d'une ou plusieurs technologies dans un établissement.

\section{Méthodologie}

10 Pour comprendre comment les enseignants et les élèves s'approprient leur ordinateur portable, nous avons mené une recherche exploratoire de type ethnographique dans deux collèges dits "expérimentaux» (ci-après dénommés $\mathrm{C} 1$ et $\mathrm{C} 2$ ). Ceux-ci ont été sélectionnés par la DANE, car les professeurs y auraient, selon elle, développé des usages pédagogiques avec les matériels informatiques dont ils ont bénéficié.

11 Si les tailles des établissements sont similaires, C1 est classé en réseau d'éducation prioritaire. Cela signifie donc qu'il possède des moyens humains et financiers supplémentaires afin de réduire l'impact que les inégalités sociales et économiques peuvent avoir sur la réussite scolaire des élèves ${ }^{5}$.

12 En ce qui concerne $\mathrm{C}$ 1, nos observations in situ se sont déroulées durant deux périodes pour «comprendre les processus, les événements et les relations dans leur contexte 
social» (Hudelson, 2004): tout d'abord, nous nous y sommes rendus de façon hebdomadaire de novembre 2013 à juin 2014, puis selon les mêmes modalités de janvier à juin 2015.

13 Le second collège, C2, a été adjoint pour nous permettre de contraster nos observations. De même que pour $\mathrm{C} 1$, nous nous y sommes rendus une fois par semaine d'avril à juin 2015.

14 Une approche qualitative a été mise en œuvre de façon inductive, c'est-à-dire "sans hypothèses de départ fortes, avec seulement un thème d'enquête, mais sans présupposés de résultats» (Alami, Desjeux, \& Garabuau-Moussaoui, 2013, paragr. 33). Dans cette perspective, un temps important a été consacré à la réalisation d'entretiens informels dans les établissements scolaires. Des entretiens semi-directifs enregistrés ont également été conduits auprès de plusieurs acteurs afin de comprendre d'une part, comment ils utilisent leur machine; et d'autre part, les effets des formes d'accompagnement sur les utilisations pédagogiques que les enseignants font avec l'Ordival. Ce sont ainsi quarantetrois entretiens qui ont été réalisés individuellement avec des professeurs. Soixante-dixhuit collégiens de $6^{\mathrm{e}}, 5^{\mathrm{e}}$ et $4^{\mathrm{e}}$ ont été rencontrés de manière individuelle ou en focus groupe. Notre objectif consistant à comprendre comment ils emploient leur ordinateur portable au collège et à la maison.

Des observations ont aussi été réalisées auprès de classes de différents niveaux allant de la $6^{\mathrm{e}}$ à la $4^{\mathrm{e}}$ dans plusieurs disciplines scolaires (anglais, éducation musicale, français, histoire-géographie, mathématiques, sciences de la vie et de la terre, technologie) de façon ponctuelle ou itérée lorsque l'Ordival était utilisé par les élèves. Nous avons cherché à comprendre comment les élèves et leur enseignant manipulaient leur poste de travail et ses ressources dans le cadre d'activités instrumentées en classe.

Il convient toutefois d'en souligner les limites. Si certaines sont propres aux recherches portant sur les $\mathrm{TIC}^{6}$, d'autres sont liées aux contextes. En effet, peu d'enseignants par discipline ont pu être interrogés et/ou observés. Chaque établissement dispose d'un professeur d'éducation musicale ou d'arts plastiques qui a en charge l'ensemble des classes de son collège. Il en va de même pour les conseillers principaux d'éducation ou les chefs d'établissements. Il nous parait donc indispensable de dire que nous ne cherchons pas à rendre compte de l'ensemble de l'opération. Les résultats obtenus sont bien entendu indicatifs et les éléments d'analyse mis en évidence ne sont pas généralisables à l'ensemble des établissements équipés en ordinateurs portables en raison de multiples différences contextuelles.

17 Par ailleurs, une approche quantitative a également été mise en œuvre deux années consécutives afin de mieux appréhender comment un échantillon d'élèves emploie cette machine dans les contextes scolaires et familiaux, et quelles en sont leurs représentations.

18 En juin 2014, nous avons diffusé aux élèves de $6^{\mathrm{e}}$ et de $5^{\mathrm{e}}$ un questionnaire réalisé sur LimeSurvey, à partir des réponses fournies par les collégiens aux entretiens exploratoires que nous avions menés en amont. La passation du questionnaire s'est déroulée, en accord avec la DANE et les chefs d'établissements des collèges où nous conduisons notre recherche, en présence d'un enseignant et de la nôtre, pendant soit une heure de permanence en salle informatique, soit une heure de cours avec l'Ordival. Nous avons ainsi récolté 432 réponses complètes. Parmi elles, $60 \%$ des élèves sont en $6^{\mathrm{e}}$ alors que 
$40 \%$ sont en $5^{\mathrm{e}}$. En outre, notre échantillon est composé de $51 \%$ de filles et $49 \%$ de garçons.

19 En juin 2015, nous avons diffusé le même questionnaire qu'en 2014, dans les mêmes conditions, auprès des élèves de $5^{\mathrm{e}}$ et $4^{\mathrm{e}}$. Notre objectif consistait à effectuer un suivi des utilisations en termes d'appropriation de l'Ordival. Lors de cette deuxième passation, nous avons obtenu 315 réponses complètes. Parmi elles, $60 \%$ des élèves sont en $5^{\mathrm{e}}$ et $40 \%$ en 4 e . Notre échantillon est semblable au premier puisqu'il est composé de $52 \%$ de filles et de $48 \%$ de garçons.

Il est un peu moins étendu qu'en 2013-2014 dans la mesure où ce sont les élèves de $6^{\mathrm{e}}$ et de $5^{\mathrm{e}}$ de quatre collèges, soit 432 collégiens, qui avaient participé à la passation du questionnaire. Malheureusement, nous ne savons pas combien de répondants de 2015 avaient répondu en 2014, ce qui nous empêche de repérer les évolutions individuelles. Malgré cela, il est possible de faire l'hypothèse d'un recouvrement non négligeable des deux échantillons.

21 Nous avons tenté de faire une comparaison des données quantitatives obtenues en 2014 et en 2015. Il convient toutefois d'en souligner les limites. D'abord, même si le questionnaire est le même et si les conditions de passation ont été harmonisées au mieux, la distribution en fonction des collèges a largement varié d'une année à l'autre. Les variations peuvent être dues au hasard, bien que rien ne le garantit. Les résultats obtenus sont donc à considérer avec beaucoup de précautions pour deux raisons. D'abord, les élèves de $6^{\mathrm{e}}$ étaient plus nombreux à répondre au questionnaire en 2014 ; les $5^{\mathrm{e}}$ l'étaient en 2015. Ensuite, bien que nous ayons souhaité interroger le même échantillon de collégiens en 2014 et en 2015, nous ne pouvons pas garantir quels sont les élèves qui y ont répondu les deux années. Il existe une cohérence globale entre les deux années.

L'analyse, effectuée sur Modalisa, donne des résultats indicatifs qui permettent de repérer des attractions entre modalités. Nous avons mis en œuvre une fonctionnalité du logiciel permettant d'effectuer des tris multiples entre une variable nominale pivot (par exemple la classe ou le genre) et un ensemble d'autres variables afin de déterminer des attractions significatives entre modalités. La force de la liaison est appréciée par le pourcentage de l'écart maximal à l'indépendance (PEM) de Cibois (1990). Nous n'avons conservé que les PEM supérieurs à $20 \%$ qui étaient significatifs au seuil de $5 \%$ (Baron, 2007).

\section{Résultats}

\subsection{Un projet fédérateur du point de vue des acteurs de direction}

L'analyse des entretiens avec les responsables montre que pour ceux de C2 tous les professeurs auraient été formés à l'utilisation de l'ordinateur portable. Selon eux, certains auraient choisi de suivre des formations pour ensuite se charger eux-mêmes de former leurs collègues. Ils se disent donc enthousiastes en raison de l'engagement et de l'investissement d'enseignants dans plusieurs projets nécessitant une utilisation pédagogique des outils numériques, et notamment de l'Ordival. Une charte numérique a donc été mise en place à la rentrée 2014-2015 pour structurer les utilisations pouvant être faites par les élèves avec les outils numériques disponibles au sein du collège. Elle définit les règles d'utilisations du matériel et d'accès aux ressources numériques. 
Des formations à destination des parents, sur le modèle du «dispositif mallette des parents $\rrbracket^{7}$, avaient été prévues en 2014-2015 afin de leur permettre d'assurer le suivi de la scolarité de leurs enfants. Une réflexion avait été également lancée sur la création d'audio-livres visant à présenter l'établissement aux futurs élèves dans le cadre de la liaison entre l'école primaire et le collège. Ces projets n'ont cependant pas été mis en œuvre par la nouvelle direction nommée à la rentrée 2015. Il leur a été substitué un nouveau projet lié au logiciel Minetest ${ }^{8}$.

Dans C1, un personnel de direction ayant pris son poste dans le collège à la rentrée 2014 déclare que plusieurs enseignants lui auraient demandé de suivre des formations sur l'utilisation des TIC. Cela témoignerait, selon lui, de l'accroissement de la volonté de l'équipe enseignante à mettre en œuvre davantage d'activités pédagogiques instrumentées, «ça commence à venir » (C1 - Chef d'établissement 1). Plusieurs projets ayant fait l'objet de demandes du pôle numérique ont conduit six enseignants à réfléchir avec leur principal adjoint à la création du site internet du collège ainsi qu'à la mise en place d'une charte d'utilisation des TIC. Ils ont été finalisés pour la rentrée 2016.

Les membres de la direction de C1 manifestent, depuis 2013, leur volonté de s'informer et disent participer à des réunions au sein de leur district. Malgré leur enthousiasme, ils font part de leur sentiment d'insécurité et se disent désireux d'avoir davantage de retours sur les expérimentations pédagogiques menées au sein de leur établissement afin de mieux évaluer les évolutions.

\subsection{Les points de vue des enseignants sur les formations}

\subsubsection{Un souhait de formation aux utilisations des équipements}

27 Parmi la soixantaine d'enseignants rencontrés, l'ensemble dit être satisfait du taux d'équipement en matériels informatiques accessibles dans leur collège. Néanmoins, des inquiétudes continuent à être exprimées par l'ensemble des enseignants en ce qui concerne le faible niveau perçu de maîtrise des outils ainsi qu'aux problèmes liés à l'infrastructure (dysfonctionnements du réseau Wifi et insuffisance des prises électriques par salles de cours). Si tous les enseignants de $\mathrm{C} 1$ font part durant nos rencontres de leur sentiment d'insécurité et se disent désireux d'être davantage formés à l'utilisation pédagogique des outils numériques dont ils bénéficient, douze expriment des besoins plus spécifiques : sept souhaiteraient être formés à l'emploi du tableau numérique interactif et cinq à la manipulation de l'Ordival et de ses logiciels.

\subsubsection{Formation continue ou accompagnement?}

solutions de formation au sein de l'établissement rencontrent un certain succès, comme le montre le nombre important d'inscrits aux modules dans l'un des collèges. La question est cependant complexe comme le suggère Baron (2014): nous notons une certaine réticence envers des contextes de formations obligatoires ou transdisciplinaires.

Par ailleurs, nos observations montrent qu'une majorité se tourne de prime abord vers leur référent numérique. La présence d'un pair, considéré comme expert par ses collègues, semble les conduire, souvent en salle des professeurs, à échanger et à tester parfois immédiatement des outils et des procédures. D'après Villemonteix, «cette modalité de formation peut constituer pour les enseignants d'une même école une 
réponse à un besoin partagé de maitrise technique et organisationnelle nécessaire au profit d'objectifs pédagogiques spécifiques »(Villemonteix, 2007, p. 152).

Comme le suggèrent Genevois et Hamon, il serait sans doute opportun de «faire évoluer les rôles des référents numériques afin que leurs tâches [soient] mieux délimitées pour éviter de les transformer en personnel de maintenance technique " (Genevois \& Hamon, 2014, p. 35). Dans ce sens, des professeurs proposent de recruter des personnels formés qui s'occuperaient exclusivement des aspects techniques afin de permettre aux référents numériques de se consacrer davantage au soutien pédagogique. Cette solution était déjà identifiée et présentée dans le rapport consacré à l'opération Ordicollège 19 (Durpaire, Jardin, Jouault, \& Perez, 2011).

\subsubsection{Les formations en dehors de l'établissement : une occasion d'échanges}

Certains répondants disent que les formations sont plutôt focalisées sur des aspects techniques et généralistes au détriment des aspects pédagogiques; pour d'autres, elles leur permettraient d'effectuer un travail sur leur représentation des TICE, ce qui pour Béziat permettrait d'opérer comme «déclencheur ou inhibiteur de l'action pour leur usage ou non-usage scolaire » (Béziat, 2012, p. 57).

Dans ce sens, une enseignante, qui fait part de son expérience au cours d'un entretien, explique avoir suivi une formation en 2014 avec le plan académique de formation (PAF). Elle lui aurait permis de découvrir, de réfléchir et d'expérimenter des ressources logicielles dans un contexte en dehors de l'espace classe avant de les utiliser avec ses élèves.

33 Ainsi, les accompagnements ont une fonction rassurante pour certains. En effet, ils leur offrent la possibilité non seulement d'expérimenter des ressources à des fins pédagogiques en dehors des murs de l'établissement, mais également d'échanger sur leur expérience avec d'autres enseignants : «ça permet de voir d'autres choses, de rencontrer d'autres gens qui des fois rencontrent les mêmes galères, de pouvoir en parler » (C1 Enseignant 1).

Cependant, une dizaine d'enseignants qui se déclarent être intéressés par des formations faites par la DANE ou le PAF, disent regretter le nombre de places limitées à ces ateliers. Ils racontent pallier à cette difficulté en visionnant des vidéos sur You Tube et en consultant des fiches pédagogiques accessibles sur des sites dédiés à leur discipline ou sur celui de la DANE. Six enseignants de C2 feraient également une veille informationnelle, par le biais de Twitter, dans le but de s'informer sur les projets pédagogiques faits par d'autres professeurs, notamment dans l'académie de Créteil.

\subsubsection{Les néo-titulaires : sensibles aux formations initiales}

Un point qu'il pourrait être intéressant d'approfondir doit être pris en compte : celui des effets de la formation initiale des néo-titulaires sur leur appropriation des équipements. Quatre révèlent ainsi avoir appris à utiliser les TIC lors d'enseignements universitaires leur étant dédiées. Bien qu'ils se disent soucieux de la préparation de leurs cours ainsi que de la gestion de leur classe, ils manifestent leur volonté d'employer les TIC dans leurs pratiques pédagogiques. Sans se dire experts dans leur utilisation, ils expliquent employer ce que Daguet appelle «des ressources prêtes à l'emploi» (Daguet, 2007, p. 6), 
c'est-à-dire, des exerciseurs tels que Labomep ou des ressources médiatiques accessibles, notamment sur You Tube.

Un professeur de mathématiques de C2 explique employer le logiciel de géométrie dynamique Géogébra découvert à l'occasion de sa préparation du certificat d'aptitude au professorat de l'enseignement du second degré (CAPES) afin d'illustrer des concepts de géométrie. Une enseignante de français de $\mathrm{C} 1$ raconte, pour sa part, introduire progressivement les TIC à ses pratiques professionnelles, notamment en faisant réfléchir ses élèves sur le lien existant entre le thème de la séquence pédagogique et des extraits filmiques ou musicaux disponibles sur You Tube.

\subsection{Les points de vue des élèves sur l'utilisation d'Ordival}

\subsubsection{Une lente appropriation des ordinateurs portables comme instruments scolaires}

Concernant les utilisations de l'Ordival pendant les cours, les réponses des $6^{\mathrm{e}}$ au questionnaire étaient en 2014 fortement centrées en technologie sur la modalité « souvent » alors que celles des $5^{\mathrm{e}}$ étaient tournées vers « jamais ». En 2015, les réponses des $5^{\mathrm{e}}$ sont orientées dans cette discipline vers les modalités "à tous les cours", « souvent » et " parfois », elles sont en revanche centrées pour les $4^{\mathrm{e}}$ sur « jamais». Cela suggère une lente « scolarisation » (Baron \& Bruillard, 2004) de l'ordinateur portable dans les pratiques scolaires des collégiens. Par ailleurs, nous observons que les élèves de $5^{\mathrm{e}}$ qui déclarent "souvent " utiliser l'Ordival dans cette discipline considèrent, de façon très significative, la machine comme "utile » et "très utile ». Il en va de même en éducation musicale où les réponses des $5^{\mathrm{e}}$ sont très significativement attirées par les modalités « à tous les cours », « souvent », et « parfois » alors qu'elles sont axées vers « jamais » en $4^{\mathrm{e}}$.

\subsubsection{Les pratiques déclarées par les élèves à la maison : des usages prescrits aux divertissements}

L'analyse des discours indique que les élèves les emploient plutôt à la maison dans l'optique de réaliser des tâches scolaires prescrites par leurs enseignants (préparer un exposé, regarder une vidéo, faire un exercice). Des logiciels découverts et utilisés pendant les cours tels que Sweet Home $3 D^{9}$ en technologie ou Minetest ${ }^{10}$ en SVT, qui sont employés pour apprendre des notions disciplinaires et/ou informatiques, sont cités à plusieurs reprises par des élèves comme des moyens de se divertir, de « jouer » à la maison.

En revanche, l'analyse des questions portant sur les façons dont les élèves emploient leur Ordival à la maison ou au collège n'offre pas la possibilité d'affirmer l'existence d'utilisations divertissantes ou d'utilisations scolairement situées. Seule une proposition a obtenu des résultats très significatifs. Il s'agit de celle portant sur le fait de naviguer sur internet dans le but de préparer un exposé. Les réponses des filles sont davantage orientées vers les modalités « à la maison et au collège » et « à la maison » alors que celles des garçons sont axées sur « tu ne fais jamais cela».

Pourtant, nous remarquons que les usages ludiques (jouer ou écouter de la musiquer par exemple) sont probablement plutôt effectués sur d'autres équipements qui semblent considérés comme "plus performant» par les élèves (ordinateur familial, tablette numérique, téléphone portable). Il y aurait donc une assimilation de l'Ordival comme un 
instrument dédié au travail scolaire qui pourrait, sans doute, s'expliquer en partie en raison des capacités techniques limitées notamment du processeur et du disque dur.

\subsubsection{Une attribution genrée des usages}

41 En cherchant à saisir les écarts existants entre les utilisations de l'Ordival et les représentations des filles ainsi que des garçons, deux éléments nous ont paru intéressants. Tout d'abord, peu de contrastes sont significatifs en ce qui concerne les utilisations scolairement situées telles que se connecter aux Cartables En Ligne ${ }^{11}$ pour consulter ses notes ou préparer un exposé. Ils indiquent néanmoins que les réponses des filles et des garçons sont plutôt attirées par la modalité « c'est autant l'un que l'autre ».

En revanche au plan des utilisations divertissantes, les résultats obtenus à la proposition « télécharger des jeux » sont très significatifs. En effet, les garçons et les filles sont attirés par la modalité "c'est les garçons qui font cela». Concernant le fait d'utiliser une messagerie instantanée pour communiquer ou encore de naviguer sur internet dans le but d'écouter de la musique ou de regarder des vidéos, les réponses des filles et des garçons sont plutôt orientées vers la modalité "c'est autant l'un que l'autre », ce qui, au regard de nos observations, traduit non seulement leur méconnaissance du vocabulaire lié à leurs activités de clavardage, mais aussi des pratiques sans distinction de genre.

\subsection{Représentations des enseignants sur les utilisations en classe d'Ordival - le pôle pédagogie}

\subsubsection{Des types d'appropriations diversifiés}

Nos observations, limitées quantitativement, suggèrent que certains ont mis en place des rituels d'installation dans l'espace de la classe des élèves avec leurs matériels. Des habitudes prises, disent-ils, dès le début de l'année faciliteraient ainsi la mise au travail des élèves dans cet espace, considéré par leurs enseignants, comme propice aux apprentissages. Certains déclarent davantage employer les ordinateurs portables avec leurs $6^{\mathrm{e}}$ qu'avec leurs autres niveaux en raison, sans doute, de ces rituels. Ils déclarent se sentir plus à l'aise pour utiliser les ordinateurs lors de séances pédagogiques avec les $6^{\mathrm{e}}$ qu'avec les autres niveaux.

Les résultats des questionnaires suggèrent une diminution des utilisations par les élèves de l'Ordival en sciences. En 2014, les $6^{\mathrm{e}}$ déclaraient ainsi l'utiliser «souvent » dans cette discipline. En 2015, les réponses des $5^{\mathrm{e}}$ sont plutôt attirées par la modalité « jamais » ou " parfois ». Cette variation pourrait s'expliquer par le fait que les personnes qui avaient la charge de l'ensemble des $6^{e}$ en 2013-2014 ne l'ont plus en 2015. Un effet enseignant semble donc prédominer.

En revanche, les réponses indiquent une augmentation des utilisations d'Ordival en éducation musicale. En effet, en 2014, les réponses des $6^{\mathrm{e}}$ étaient attirées par la modalité « jamais »; en 2015, elles sont plutôt centrées vers « parfois » et " souvent ». Cela pourrait être expliqué dans C2 par la mise en œuvre de projets didactiques, initiés par des enseignants et formalisés durant des conseils pédagogiques, qui nécessitent l'emploi de l'Ordival dans un niveau donné (les $5^{\mathrm{e}}$ ). 
Dans leurs discours, ils expriment à ce propos deux points intéressants qui mériteraient d'être approfondis dans la mesure où ils semblent impacter les usages pouvant être faits avec les machines :

- la méfiance des professeurs vis-à-vis des équipements informatiques qu'ils déclarent être anciens et plus lents, ce qui nous amène à nous questionner sur leur représentation d'une obsolescence du matériel informatique ;

- la représentation des enseignants d'une plus grande maniabilité des élèves de $6^{e}$.

\subsubsection{Impacts possibles des formations sur les conceptions de l'enseignement et de l'apprentissage des enseignants}

Certains enseignants disent avoir bénéficié de formes d'accompagnement qui leur auraient également permis de développer deux types d'utilisation dans le contexte de la classe. Cela fait écho aux recherches de Villemonteix et Béziat sur la culture technique et la formation des enseignants du primaire. D'après eux, il y aurait d'une part, une minorité d'enseignants qui se sentirait "à l'aise techniquement »; d'autre part, une majorité, qui aurait le sentiment d'être en danger et de manquer d'expérience, ce qui expliquerait les utilisations pédagogiques effectuées ou non avec les technologies (Villemonteix \& Béziat, 2014).

- Des utilisations à des moments précis du programme, déterminées en amont par l'enseignant. L'ordinateur portable peut être utilisé pour travailler sur un point précis du programme et pour permettre aux élèves de développer plusieurs compétences, notamment en expression orale par le biais de l'utilisation de logiciels tels que Audacity, ou en expression écrite en utilisant, par exemple, le logiciel de traitement de texte. Dans ces cas de figure, des professeurs observés déclarent avoir préparé leur séance en amont, soit pendant une formation, soit avec l'assistance du référent numérique.

- Des utilisations en co-animation avec un pair. Nous avons observé que des séances pouvaient être réalisées en co-animation avec un pair ; c'est-à-dire, dans les cas observés, par le référent numérique de l'établissement. Une enseignante, qui se déclarait « anxieuse » du déroulement de sa séance, dit se sentir rassurée de la présence du référent numérique qu'elle dit être meilleure qu'[elle] en informatique.

\subsubsection{Des objectifs pédagogiques diversifiés pour l'utilisation d'Ordival}

Les utilisations en co-animation donneraient la possibilité aux enseignants d'avoir une approche plus individualisée et tendraient à favoriser la prise en main des outils manipulés. Des enseignants disent, à ce propos, pouvoir davantage se concentrer sur chaque apprenant, ce qui nous paraît faire écho à une recherche menée par Baron et Daguet dans laquelle ils soulignent «le rôle central de l'apprenant dans le processus d'enseignement et le rôle de tuteur, d'accompagnateur, d'accoucheur, ou encore [...] de coach que l'enseignant est amené à jouer » (Baron \& Daguet, 2005, paragr. 22).

Nos observations, bien que limitées quantitativement là encore, donnent quelques éléments de réflexion qu'il serait intéressant d'approfondir sur les effets que l'utilisation des machines pourrait avoir sur l'apprentissage des élèves.

Tout d'abord, deux enseignants de technologie disent avoir fait utiliser le logiciel Sweet Home 3D afin que leurs élèves conçoivent numériquement des habitats. Nous avons observé que l'une enseignante d'entre eux a ainsi fait réaliser à ses élèves de $5^{\mathrm{e}}$ des immeubles. Cela les aurait conduits à développer des compétences en informatique. 
51 Deux élèves observés nous ont expliqué pendant une récréation, durant laquelle leur enseignante leur a permis de continuer leur activité, qu'ils s'interrogeaient sur les limites du logiciel. Ils ont fait l'hypothèse qu'il n'était pas possible de créer plus de quatorze étages à leurs immeubles. Ils sont alors entrés dans une posture de recherche, et ont testé leur hypothèse de départ.

52 Ensuite, deux enseignants de mathématiques nous ont expliqué, pendant des entretiens, faire utiliser le jeu sérieux Table en poche ${ }^{12}$ ainsi que la plate-forme Labomep afin de permettre à leurs élèves de développer des compétences en calcul mental. Ils auraient remarqué que certains élèves en difficulté s'exerçaient chez eux en faisant des exercices, notamment sur cette plate-forme.

53 Par ailleurs, nous avons pu observer que certains élèves se connectaient, après avoir terminé un exercice prescrit par leur enseignant, sur cette plate-forme pour se divertir, sans que cela ne leur soit demandé. Nous avons remarqué que des camarades à proximité s'y connectaient, sans doute, par effet d'émulation.

54 Enfin, les observations réalisées indiquent que les élèves sont amenés à développer des compétences en expression orale et écrite par l'emploi de logiciels tels qu'Audacity ou LibreOffice Writer utilisés dans plusieurs disciplines (anglais, français, éducation musicale, histoire-géographie). Audacity amènerait les élèves de quatre classes de sixième et une de cinquième à augmenter leur temps de parole et ainsi à s'exercer à l'oral (CombemorelPauty, 2016).

\section{Discussion et perspectives}

Nous avons mené notre recherche dans deux collèges a priori réputés, par les collectivités territoriales dont ils dépendent, pour leur utilisation des technologies numériques. Les entretiens et les observations réalisés montrent qu'il y a peu d'usages des ordinateurs portables en classe. En général, ils sont plutôt ponctuels sauf dans le cas particulier de personnes particulièrement motivées. Ils dépendent des objectifs pédagogiques des enseignants.

56 Cette situation pourrait s'expliquer, d'une part, par les politiques éducatives mises en œuvre par le pôle numérique pour favoriser l'appropriation des ordinateurs, ce qui suggère que les améliorations proposées notamment dans le rapport consacré au plan Ordicollège (Durpaire et al., 2011) ont été prises en compte. D'autre part, par un nombre important d'enseignants qui ont suivi des formations en dehors de leur collège avant de devenir, à leur tour, accompagnateurs au sein de leur établissement, ce qui pourrait être un vecteur d'appropriation des technologies numériques.

57 Ces résultats confirment ceux obtenus par Khaneboubi (2009a): les professeurs qui emploient l'Ordival en cours sont souvent ceux qui enseignent une discipline historiquement liée aux usages des TIC (arts plastiques, éducation musicale, mathématiques).

58 L'analyse des discours des acteurs des établissements nous a permis de constater de micro-évolutions au plan des pratiques numériques, ce qui est confirmé par l'analyse statistique des questionnaires diffusés en 2014 et en 2015. Des professeurs qui disaient en 2014 ne pas avoir intégré les équipements dans leurs pratiques pédagogiques en raison d'un manque d'expérience, ont découvert à la suite d'échanges en salle des enseignants, sur leur temps libre, des ressources logicielles accessibles dans leur machine. Ces derniers 
déclarent, en 2015, être désireux de se sentir à l'aise avec les outils technologiques avant de les intégrer dans leur classe. D'autres ont mis en œuvre des projets culturels avec des enseignants issus de traditions disciplinaires différentes avec d'autres types d'équipements, les tablettes numériques. Celles-ci ont été introduites pendant l'année scolaire 2013-2014 dans l'un des établissements par le conseil général et dans l'autre par le CANOPÉ.

Plusieurs enseignants, principalement d'Éducation physique et sportive et d'arts plastiques, qui disaient avoir accueilli favorablement les tablettes lors de leur implantation, se sont investis dans des projets pédagogiques les nécessitant, peut-être, au détriment de leur appropriation de l'ordival. Ils ont ainsi mis en œuvre des scénarios pédagogiques où les applications multimédias (photos, vidéos, sons) sont privilégiées, ce qui les a conduits à modifier leur approche de façon innovante au plan de la gestion de la classe. Une enseignante d'arts plastiques a réagencé l'espace classe en îlot pour permettre un enseignement moins frontal et un apprentissage plus collaboratif.

Malgré la perception d'une progression au plan des utilisations des Ordivals dans le contexte de la classe par certains enseignants, il est donc nécessaire de rappeler que la scolarisation des technologies de l'information et de la communication relève d'un «lent » processus (Baron, 2005). De ce fait, il serait intéressant de continuer l'analyse sur une durée plus importante pour comprendre les effets des formes d'accompagnement mises en œuvre par les politiques éducatives locales sur la manière dont les enseignants emploient les outils et les ressources numériques à leur disposition pour enseigner ainsi que sur les apprentissages des élèves.

Bien qu'il reste à ce jour encore des résultats à tirer des questions ouvertes du questionnaire, nous faisons l'hypothèse que lorsque les élèves considèrent qu'il est utile, voire très utile, d'employer l'Ordival dans une discipline, ils le justifient notamment par des utilisations déjà faites pendant les temps de classe avec leurs enseignants. Il en irait de même pour les questions relatives aux logiciels et sites utilisés.

Pour l'avenir, deux pistes nous semblent intéressantes.

63 S'intéresser aux enseignants. Les entretiens et les observations réalisés jusqu'ici indiquent que ces derniers utilisent les Ordivals davantage avec les élèves de $6^{\mathrm{e}}$ qu'avec les autres niveaux, ce qui pourrait être un facteur, à prendre en compte expliquant le faible taux d'utilisation déclaré par l'échantillon d'élèves interrogés.

- Il serait également intéressant de s'intéresser à la manière dont les enseignants produisent, modifient et disséminent des ressources, notamment en relation avec les formations dispensées par le PAF et par la DANE. Quels réseaux d'échange en ligne, plus ou moins informels se créent et se développent, comment contribuent-ils à l'appropriation par les enseignants de pratiques instrumentées en classe?

- Étudier les activités des élèves. Comment évoluent les pratiques juvéniles avec les technologies numériques en fonction de leurs âges et du groupe de pairs qui les environne? Comment construisent-ils des cultures numériques? Deux hypothèses pourraient être testées afin d'approfondir cette idée :

- Une forme de «tyrannie de la majorité » (Pasquier, 2005) s'exercerait sur les jeunes et les amènerait à développer, par essaimage et sans distinction de genre, les mêmes types d'usages que leurs pairs avec les technologies numériques.

- Des activités instrumentées faites dans la sphère scolaire permettraient aux élèves de s'approprier la machine et de développer une culture numérique sans distinction de 
genre, ce qui conduirait à une évolution progressive des représentations, notamment des stéréotypes de genre.

Finalement, bien d'autres pistes sont sans doute ouvertes, en relation avec le lancement en 2014 du plan de refondation de l'École initié par François Hollande. Une attention plus particulière pourra être portée à la manière dont les collégiens dotés de l'Ordival sont accompagnés par leurs enseignants à la découverte et l'appropriation des environnements techniques ainsi qu'à la compréhension des concepts informatiques afférents, notamment dans le cadre des activités proposées par la plate-forme d'évaluation et de certification des compétences numériques nommée PIX ${ }^{13}$.

\section{BIBLIOGRAPHIE}

Alami, S., Desjeux, D., \& Garabuau-Moussaoui, I. (2013). L'approche qualitative. Que sais-je ?, 2e éd., 11-29. Consulté à l'adresse https://www-cairn-info.frodon.univ-paris5.fr/resume.php ? ID_ARTICLE =PUF_ALAMI_2013_01_0011

Baron, G.-L. (2005). Les TICE, de l'innovation à la scolarisation. Problèmes et perspectives (p. 11). Présenté à Accompagner les TICE à l'École. Consulté à l'adresse http://aft-rn.net/actes_colloque05/ conferences/conference_GL_Baron.pdf

Baron, G.-L. (2007). À propos d'un indicateur de liaison entre les modalités d'un tableau de contingence, le PEM [Université Paris Descartes]. Consulté 23 mars 2016, à l'adresse http:// blogs.parisdescartes.fr/glbaron/weblog/1913.html

Baron, G.-L. (2014, septembre 9). Numérique et formation des enseignants du primaire en contexte francophone. Éléments de questionnement [Université Paris Descartes]. Consulté 13 septembre 2014, à l'adresse http://carnets.parisdescartes.fr/blog/view/157811/numerique-etformation-des-enseignants-du-primaire-en-contexte-francophone-elements-de-questionnement Baron, G.-L., \& Bruillard, E. (2004). Quelques réflexions autour des phénomènes de scolarisation des technologies. In Entre technique et pédagogie. La création de contenus multimédia pour l'enseignement et la formation (p. 154-161). Neuchâtel. Consulté à l'adresse http://tinyurl.com/ nhqsogw

Baron, G.-L., \& Combemorel-Pauty, C. (2015). Rapport de synthèse de la recherche sur le projet Ordival. Baron, G.-L., \& Daguet, H. (2005). De l'innovation à la scolarisation. Le cas des technologies de l'information et de la communication. Connaissances de la diversité, 233-243. Consulté à l'adresse http://www.cairn.info/article.php?ID_ARTICLE=ERES_TALBO_2005_01_0233

Baron, G.-L., Voulgre, E., Khaneboubi, M., Zablot, S., \& Combemorel-Pauty, C. (2014). Rapport technique numéro 2 de la recherche ORDIVAL (Rapport de recherche) (p. 43). Paris : Université Paris Descartes. Consulté à l'adresse http://gl.baron.free.fr/wagons/ordival_juillet14.pdf

Battaglia, M. (2014, novembre 6). François Hollande annonce « un grand plan numérique pour l'école ». Le Monde.fr. Consulté à l'adresse http://www.lemonde.fr/ecole-primaire-et-secondaire/ article/2014/11/06/francois-hollande-annonce-un-grand-plan-numerique-pour-lecole_4519897_1473688.html 
Béziat, J. (2012). Former aux TICE : entre compétences techniques et modèles pédagogiques. Revue internationale des technologies en pédagogie universitaire, 9(1-2), 53. https:// doi.org/10.7202/1012902ar

Cibois, P. (1990). L’Analyse des données en sociologie. Paris : Presses universitaires de France. 1990.

Combemorel-Pauty, C. (2016). Transmettre une culture numérique à des élèves équipés en ordinateurs portables par un département : le cas de l'utilisation du logiciel Audacity. In Quelles éducations au numérique, en classe et pour la vie ? Namur. Consulté à l'adresse http:// didapro6.sciencesconf.org/87558/document

Daguet, H. (2007). Vers une catégorisation des usages TICE des enseignants. Actualité de la Recherche en Éducation et en Formation. Consulté à l'adresse http://www.congresintaref.org/ actes_pdf/AREF2007_Herve_DAGUET_530.pdf

Durpaire, J.-L., Jardin, J., Jouault, D., \& Perez, M. (2011). Le plan Ordicollège dans le département de la Corrèze (IGEN No. 2011-112). Ministère de l'éducation nationale, de la jeunesse et de la vie associative. Consulté à l'adresse http://www.education.gouv.fr/cid67068/le-plan-ordicollegedans-le-departement-de-la-correze.html

Genevois, S., \& Hamon, D. (2014). ENT 93 - Rapport de recherche sur les usages des ENT dans les collèges de Seine-Saint-Denis (report). Consulté à l'adresse https://hal.archives-ouvertes.fr/hal-00991475/ document

Hudelson, P. (2004). ROCARE : Extraits de guides pour la Recherche Qualitative. Consulté 10 février 2016, à l'adresse http://www.ernwaca.org/panaf/RQ/fr/annexea.php

IGEN. (2011). Le plan Ordicollège dans le département de la Corrèze (No. 2011-112). Consulté à l'adresse http://www.ordicollege.cg19.fr/pdfs/rapport_igen.pdf

Jaillet, A. (2004). What Is Happening with Portable Computers in Schools? Journal of Science Education and Technology, 13(1), 115-128.

Khaneboubi, M. (2007). Usages de l'informatique au collège et habitus professionnels des enseignants : exemple de l'opération « un collégien, un ordinateur portable » dans le département des Landes. Bordeaux 2. Consulté à l'adresse http://www.theses.fr/2007BOR21432

Khaneboubi, M. (2009a). Description de quelques caractéristiques communes aux opérations de dotations massives en ordinateurs portables en France. Sciences et Technologies de l'Information et de la Communication pour l'Éducation et la Formation, 16, 8 pages. Consulté à l'adresse https:// hal.archives-ouvertes.fr/hal-00696410

Khaneboubi, M. (2009b). Facteurs influençant les usages de l'informatique en classe par des enseignants de collèges du département des Landes. In G.-L. Baron, E. Bruillard, \& L.-O. Pochon, Informatique et progiciels en éducation et en formation : continuités et perspectives (p. 154-167). Consulté à l'adresse https://edutice.archives-ouvertes.fr/edutice-00765423/document Liautard, D. (2007). Bilan des opérations « Portables ». Propos de bilan... À propos d'Ordina 13. Les dossiers de l'ingénierie éducative. Consulté à l'adresse http://www.cndp.fr/archivage/ valid/93855/93855-15525-19513.pdf

Pasquier, D. (2005). Cultures lycéennes : la tyrannie de la majorité. Paris : Éditions Autrement. DL 2005 , cop. 2005.

Peraya, D. (1999). Vers les campus virtuels. Principes et fondements techno-sémio-pragmatiques des dispositifs de formation virtuels. Consulté à l'adresse https://tinyurl.com/jgwwvud 
Plantard, P. (2015). Contre la « fracture numérique ", pas de coup de tablette magique ! Projet, 345(2), 23. https://doi.org/10.3917/pro.345.0023

Rinaudo, J.-L. (2007). Des ordinateurs à bonne distance. Empan, $n^{\circ}$ 66(2), 133-137. Consulté à l'adresse https://www.cairn.info/article.php ?ID_ARTICLE =EMPA_066_0133

Rinaudo, J.-L., Turban, J.-M., Delande, P., \& Ohana, D. (2008). Des ordinateurs portables, des collégiens, des professeurs, des parents : rapport de recherche sur le dispositif Ordi 35 2005-2007. Consulté à l'adresse http://www.marsouin.org/IMG/pdf/ordi_35_RAPPORT.pdf

Villemonteix, F. (2007). Les animateurs TICE à l'école primaire : spécificités et devenir d'un groupe professionnel. Analyse de processus de professionnalisation dans une communauté de pratiques en ligne - document. Paris 5. Consulté à l'adresse https://tel.archives-ouvertes.fr/ tel-00198063/document

Villemonteix, F., \& Béziat, J. (2014). Le TNI à l'école primaire : entre contraintes et engagement, STICEF, 20 Consulté à l'adresse http://sticef.univ-lemans.fr/num/vol2013/17-villemonteix-reiah/ sticef_2013_NS_villemonteix_17.htm

Voulgre, E. (2011). Une approche systémique des TICE dans le système scolaire français : entre finalités prescrites, ressources et usages par les enseignants. Consulté à l'adresse http://shs-app.univ-rouen.fr/ civiic/memoires_theses/textes/these_VOULGRE.pdf

Wallet, J. (2010). Technologie et gouvernance des systèmes éducatifs. In Apprendre avec les technologies (p. 71-80).

\section{NOTES}

1. https://www.valdemarne.fr/a-votre-service/education/ordival consulté le 02/12/17;

2. http://docplayer.fr/2660598-Le-departement-remet-a-tous-les-collegiens-unordinateur-portable-ordival-d-emploi-mode-parents.html consulté le 22/03/16

3. http://edumarket.crdp-creteil.fr/node/1753 consulté le 10/02/16

4. http://mediafiches.ac-creteil.fr/ consulté le 15/02/16

5. http://www.education.gouv.fr/cid187/l-educationprioritaire.html\#L_education_prioritaire_a_la_rentree_2015 consulté le 11/02/16 6. Seule vingt-cinq enseignants sur la soixantaine en exercice dans les deux collèges nous a permis d'effectuer des observations dans leur classe et ce, principalement quand l'Ordival, objet de notre recherche, était employé.

7. L'objectif de ce dispositif conçu par le Ministère de l'Éducation Nationale consiste à développer un dialogue entre les parents d'élèves et l'École à travers des ateliers. D'après http://eduscol.education.fr/cid53343/mallette-des-parents-6eme.html consulté le $15 / 02 / 16$

8. Minetest est un jeu vidéo dont la licence est libre. Il figure parmi les ressources matricielles recensées par le pôle numérique. Il est téléchargeable via la Médiathèque en ligne.

9. Sweet Home 3D est un logiciel libre qui permet de concevoir son aménagement intérieur, en dessinant notamment les plans d'une habitation et en y plaçant des meubles. D'après http://www.sweethome3d.com/fr/ consulté le 24/02/16.

10. Minetest est un logiciel libre de type bac à sable inspiré de Minecraft. Il offre la possibilité de construire un monde en cubes. D'après http://www.minetest.net/ consulté le $24 / 02 / 16$. 
11. Le Cartable En Ligne est l'environnement numérique de travail déployé depuis 2007 par l'académie de Créteil.

12. Ce jeu est accessible librement sur le site Mathenpoche de Sésamath à l'adresse suivante : http://mathenpoche.sesamath.net/ceintures/calculatice/asteroideparakidi.swf . Son objectif consiste à obtenir le meilleur score possible en écrivant les résultats d'opérations avant qu'elles n'atteignent le bas de l'écran et ce, sans prendre en compte leur ordre d'arrivée.

13. Voir https://pix.beta.gouv.fr/

\section{RÉSUMÉS}

Cet article présente les premiers résultats d'une recherche en cours, conduite depuis 2013 et centrée sur les trajectoires d'appropriation d'enseignants et d'élèves équipés en ordinateurs portables par des autorités locales près de la banlieue parisienne. Cette recherche qui est principalement d'orientation ethnographique utilise aussi des outils quantitatifs tels que des questionnaires. Nos résultats montrent que les élèves n'ont pas d'usage intensif de leurs ordinateurs portables pour le travail scolaire en classe. Cependant, il y a une évolution manifeste de leurs usages, en particulier dans certaines disciplines scolaires. Cela peut être expliqué par deux facteurs principaux: le premier est lié aux politiques éducatives mises en œuvre pour favoriser l'utilisation des TIC dans les classes. L'autre est l'existence d'un processus durant lequel les enseignants suivent des formations en dehors de l'école, parfois de façon informelle, pour plus tard accompagner à leur tour leurs collègues.

This paper presents the first results of an ongoing research, led since 2013 and focusing on the appropriation path followed by teachers and students regarding laptops provided to junior high schools by local authorities in a suburb of Paris. This research has mainly an ethnographic orientation but also uses quantitative tools like questionnaires. Our results show that students do not intensively use their laptops for classroom work, but evolution is manifest, in some subject matters. This may be explained by two main factors : the first one is linked to the educational policies implemented to foster ICT use in classrooms. The other is the existence of a process where teachers follow professional development outside of the school, sometimes on an informal basis and later are able to accompany their colleagues.

\section{INDEX}

Keywords : appropriation, digital culture, pupils, teachers, laptops

Mots-clés : appropriation, culture numérique, élèves, enseignants, ordinateurs portables

\section{AUTEURS}

\section{CHRISTELLE PAUTY-COMBEMOREL}

Doctorante en sciences de l'éducation, Université Paris-Descartes, Laboratoire EDA 


\section{GEORGES-LOUIS BARON}

Professeur émérite de sciences de l'éducation, Université Paris Descartes, Laboratoire EDA 